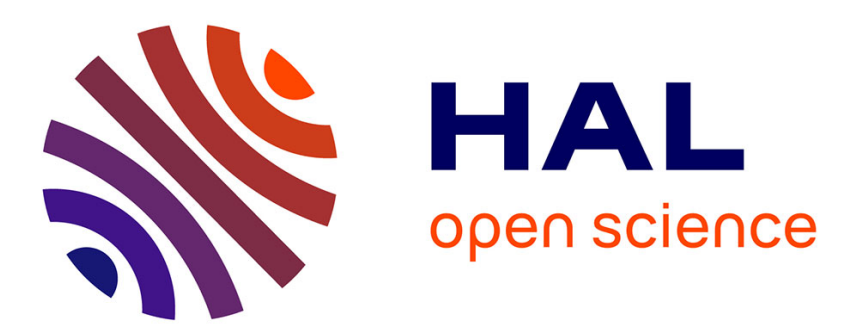

\title{
Stress-Based Model for the Breathing of Metal-Organic Frameworks
}

Alexander V Neimark, François-Xavier Coudert, Anne Boutin, Alain H. Fuchs

\section{To cite this version:}

Alexander V Neimark, François-Xavier Coudert, Anne Boutin, Alain H. Fuchs. Stress-Based Model for the Breathing of Metal-Organic Frameworks. Journal of Physical Chemistry Letters, 2009, 1 (1), pp.445-449. 10.1021/jz9003087 . hal-00548069

\section{HAL Id: hal-00548069 \\ https://hal.science/hal-00548069}

Submitted on 21 Apr 2019

HAL is a multi-disciplinary open access archive for the deposit and dissemination of scientific research documents, whether they are published or not. The documents may come from teaching and research institutions in France or abroad, or from public or private research centers.
L'archive ouverte pluridisciplinaire HAL, est destinée au dépôt et à la diffusion de documents scientifiques de niveau recherche, publiés ou non, émanant des établissements d'enseignement et de recherche français ou étrangers, des laboratoires publics ou privés. 


\section{Stress-based model for the breathing}

\section{of metal-organic frameworks}

Alexander V. Neimark, ${ }^{*, a, b}$, François-Xavier Coudert, ${ }^{* a}$ Anne Boutin ${ }^{c}$ Alain H. Fuchs ${ }^{a}$

Chimie ParisTech (École nationale supérieure de chimie de Paris), CNRS and Univ. Pierre et Marie Curie, 11 rue Pierre et Marie Curie, 75005 Paris, France.

Department of Chemical and Biochemical Engineering, Rutgers, The State University of New Jersey, 98

Brett Road, Piscataway, New Jersey 08854-8058, USA.

Chemistry Department, École normale supérieure, 24 rue Lhomond, 75005 Paris, France.

AUTHOR EMAIL ADDRESS: aneimark@rutgers.edu,fx.coudert@ chimie-paristech.fr

${ }^{a}$ Chimie ParisTech, CNRS and Univ. Pierre et Marie Curie.

${ }^{\mathrm{b}}$ Rutgers, The State University of New Jersey.

${ }^{\mathrm{c}}$ École normale supérieure. 
ABSTRACT: Gas adsorption in pores of flexible metal-organic frameworks (MOF) induces elastic deformation and structural transitions associated with stepwise expansion and contraction of the material, known as breathing transitions between large pore (lp) and narrow pore (np) phases. We present here a simple yet instructive model for the physical mechanism of this enigmatic phenomenon considering the adsorption-induced stress exerted on the material as a stimulus that triggers breathing transitions. The proposed model implies that the structural transitions in MOFs occur when the stress reaches a certain critical threshold. We showcase this model drawing on the example of Xe adsorption on MIL-53 (Al) at $220 \mathrm{~K}$, which exhibits two consecutive hysteretic breathing transitions between lp and $\mathbf{n p}$ phases. We also propose an explanation for the experimentally observed coexistence of $\mathbf{n p}$ and Ip phases in MIL-53 materials.

KEYWORDS: MOF, metal-organic frameworks, MIL-53, adsorption, structural flexibility, adsorption stress.

\section{TOC graphic.}

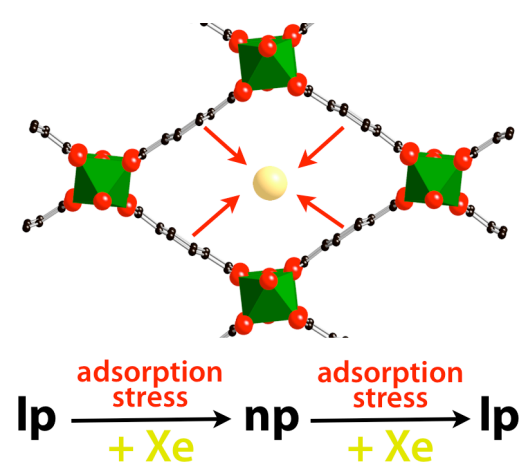


Metal-organic frameworks (MOFs) represent a rapidly expanding class of nanoporous materials that display an extremely wide range of crystal structures and host-guest properties due to the tunable porosity allowed by coordination chemistry, and the versatility enabled by functionalisation of organic linkers. It is anticipated that MOFs may have a major impact in many areas of science and technology. ${ }^{1}$ A number of these materials exhibit an exceptional flexibility and stimulus-responsive behavior, ${ }^{2,3,4,5,6}$ reacting to changes in temperature, pressure, and adsorption of guest molecules by undergoing structural transformations. Such materials have promising applications as sensors and actuators, as well as in adsorptive separation. ${ }^{3,5,7}$ The stimulus-responsive MOFs can be classified in two distinct categories. In some cases, variations in structure are progressive, as is the case of the swelling of MIL-88 upon exposure to water and various alcohols. ${ }^{8}$ In other materials, the structural response is displayed by an abrupt transition between two distinct structures of the framework. A particularly remarkable example of MOFs exhibiting this bistability is the MIL-53 materials family, ${ }^{9,10,11}$ which have recently attracted a lot of attention due to their prominent flexibility and the occurrence of a double, guest-induced structural transition (“breathing”) upon adsorption of some gases (see Fig. 1). 

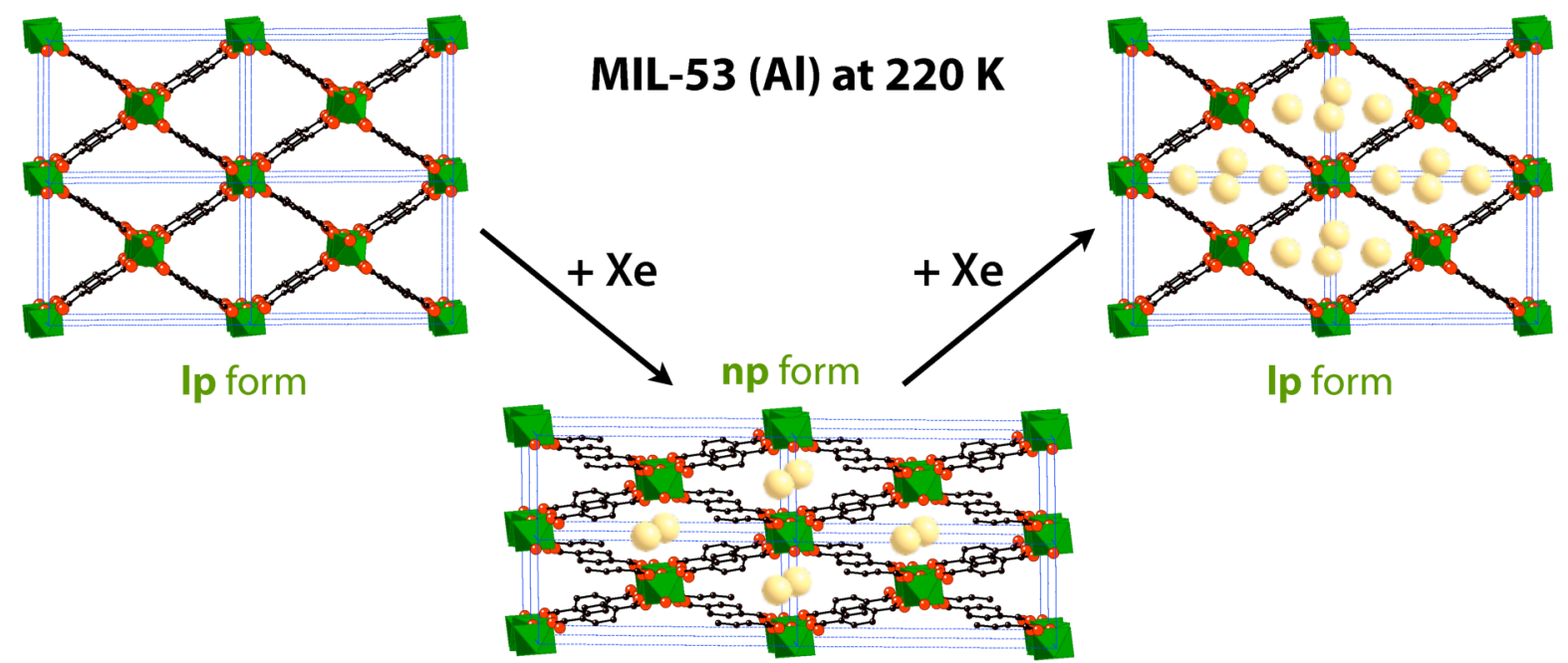

Figure 1. Schematic representation of the breathing phenomenon of MIL-53 (Al), exhibiting two successive structural transitions upon adsorption of some gases (here, Xe at $220 \mathrm{~K}$ ). The two possible states of bistable MIL-53 (Al) are called $\mathbf{n p}$ (narrow pore) and lp (large pore).

The MIL-53 framework is made of parallel one-dimensional $\mathrm{M}(\mathrm{OH})$ chains $\left(\mathrm{M}=\mathrm{Al}^{3+}, \mathrm{Cr}^{3+}\right)$, linked together by 1,4-benzenedicarboxylate (BDC) ligands to form linear diamond-shaped channels that are wide enough to accommodate small guest molecules. This structure may oscillate (or "breathe") between two distinct conformations called a large-pore phase (lp) and a narrow-pore phase (np; see Fig. 1), which have a remarkable difference in cell volume of up to $40 \%$. This breathing is clearly displayed on the adsorption-desorption isotherm of Xe at $220 \mathrm{~K}$ with two distinct hysteresis loops (Fig. 2). ${ }^{12}$ At room temperature and in the absence of guest molecules, Ip phase is the most stable form. ${ }^{6}$ However in the course of Xe adsorption, lp phase is transformed into np phase at low pressures and the reverse transformation occurs at high pressures. The conditions, at which the breathing of MIL-53 happens, have been widely studied earlier, and it was shown in particular to be triggered by changes in temperature, ${ }^{6}$ as well as adsorption of some gases and gas mixtures, but not others. ${ }^{11,13}$ Recent work, that considered the issue from the point of view of thermodynamics, has been successful in rationalizing the conditions for the occurrence of breathing in MIL-53. ${ }^{13}$ It was shown that, upon gas adsorption at a given temperature, the occurrence of breathing is conditioned by the relative adsorption affinities of the 
gas for the two host phases, lp and $\mathbf{n p}$, measured by the ratio of the Henry constants, $K_{\mathrm{H}}^{\text {lp }} / K_{\mathrm{H}}^{\text {np }}$, and by the intrinsic stability of the two respective framework conformations, characterized by the free energy difference $\Delta F_{\text {host }}$ between the $\mathbf{l p}$ and $\mathbf{n p}$ phases. ${ }^{14}$ This thermodynamic analysis was then extended to fluid mixtures ${ }^{15}$ and to an investigation of temperature dependence, which allowed to predict complete pressure-composition and pressure-temperature phase diagrams for various adsorbates in MIL-53. ${ }^{12,15}$

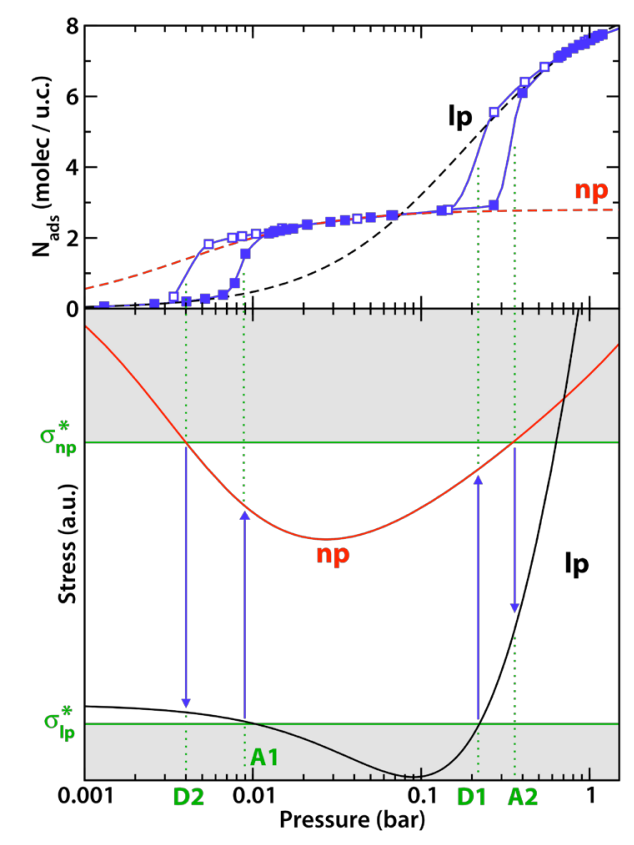

Figure 2. Upper panel: experimental adsorption and desorption isotherms of Xe in MIL-53 (Al) at $220 \mathrm{~K}^{12}$ (in blue), in logarithmic pressure scale, along with Langmuirian fits of the parts of the isotherm corresponding to the lp and np structures (black and red). Lower panel: adsorption stress for both host phases (black: lp; red: np) and critical stress $\sigma_{\mathbf{n p}}^{*}$ and $\sigma_{\mathbf{l p}}^{*}$ determining the structural transitions upon adsorption and desorption (blue arrows, and pressures noted as A1, A2, D1 and D2).

The thermodynamic approach described above is useful in understanding whether breathing occurs or not and in calculating the equilibrium vapor pressures, at which the structural transitions may take place. However, it makes no assumption about and gives no insight into physical mechanisms of structural transitions associated with breathing, nor does it explain the hysteresis loops experimentally observed. 
Numerous structural chemistry studies reported on MIL-53 are extremely useful for a general understanding of the phenomenon, but provide no direct insight into the transition mechanisms. ${ }^{3}$ In this letter, we present a simple yet instructive model for the structural transformations undergone by MIL-53 upon gas adsorption, considering the adsorption-induced stress as a stimulus that triggers these transformations. We describe how this model inherently accounts for the adsorption hysteresis observed experimentally and showcase, drawing on the example of Xenon adsorption in MIL-53 (Al) at $220 \mathrm{~K},{ }^{12}$ that the model is coherent with the experimental data available so far. Finally, we propose an explanation for the existence of mixtures of $\mathbf{n p}$ and $\mathbf{l p}$ phases observed in XRD experiments of $\mathrm{CO}_{2}$ adsorption in MIL-53. ${ }^{16}$

The proposed model relates the stress, exerted by the adsorbed phase on the adsorbent framework, with the adsorption isotherm. From the thermodynamic standpoint, the adsorption stress $\sigma_{s}$ can be quantified by derivative of the grand thermodynamic potential $\Omega_{\mathrm{c}}$ of the adsorbed phase per unit cell with respect to the unit cell volume $V_{\mathrm{c}}$ at fixed temperature $T$ and adsorbate chemical potential $\mu,{ }^{17,18}$

$$
\sigma_{s}\left(V_{c}\right)=-\left.\left(\frac{\partial \Omega_{c}}{\partial V_{c}}\right)\right|_{\mu, T}
$$

In pores of simple geometries of slit, cylindrical, or spherical shapes, the adsorption stress has a simple physical interpretation as a normal to the pore wall component of the stress tensor in the adsorbed phase. ${ }^{19,20,21}$ In anisotropic materials like MOFs this interpretation is no longer valid, and one needs to introduce tensor quantities. However, the adsorption stress defined by Eq. 1 can serve as an overall scalar measure of the magnitude of the adsorption forces acting on the porous framework. The difference between the adsorption stress, $\sigma_{s}$, and the external pressure represents the so-called solvation or disjoining pressure, $P_{s}$, which determines the magnitude of framework elastic deformation in terms of the volumetric strain $\varepsilon\left(\varepsilon=\Delta V_{\mathrm{c}} / V_{\mathrm{c}}\right.$, where $\Delta V_{\mathrm{c}}$ is the variation of the cell volume), assuming the linear Hooke law with an effective framework bulk modulus $K, P_{s}=\sigma_{s}-p_{\text {ext }}=K \varepsilon+\sigma_{0}$, where $\sigma_{0}$ is a prestress in the reference state at which the cell volume $V_{\mathrm{c}}$ is defined. ${ }^{17}$ 
The linear elasticity theory describes adsorption-induced deformations of microporous materials like zeolites and activated carbons, when the strain is small, typically in fractions of percent. ${ }^{22}$. For breathing MOFs, experiencing structural transitions with the volume changes in tens of percent, the stress-strain linearity should hold only for the stable lp and np phases. In the vicinity of the transition, the stressstrain relationship becomes necessarily nonlinear and it may even diverge at the onset of the transition. We hypothesize that the structural transition occurs when the solvation pressure approaches a certain critical stress $\sigma^{*}$ that the framework cannot resist. The critical stress $\sigma_{\text {lp }}^{*}$ associated with the lp $\rightarrow \mathbf{n p}$ transitions should be negative, because this transition corresponds to a framework contraction, while the critical stress of $\mathbf{l p} \rightarrow \mathbf{n p}$ transition $\sigma^{*}{ }_{\mathbf{n p}}$ should be positive. As shown below, this hypothesis explains the hysteric behavior of structural transitions in breathing MOFs and is consistent with the existence of two breathing transitions in MIL-53 at low and high adsorptive pressures.

As shown earlier, the adsorption isotherms of Xe and some other vapors in lp and $\mathbf{n p}$ structures of MIL-53 can be described by Langmuir equations with reasonable accuracy. ${ }^{12,13,14}$ The Xe isotherm, plotted in Fig. 2 (upper panel) in logarithmic pressure coordinates, fits two Langmuirian isotherms for lp and np structures. These isotherms are presented by broken lines. It is remarkable that the same Langmuirian isotherm adequately describes the adsorption in lp structure at low pressures prior to the $\mathbf{l p} \rightarrow \mathbf{n p}$ transition and adsorption at high pressures after $\mathbf{n p} \rightarrow \mathbf{l p}$ transition. This observation complies with a picture of Xe adsorption in MIL-53 as a homogeneous filling of the pore channels, without distinctive, preferential adsorption sites. The saturation uptakes of Xe at $220 \mathrm{~K}$ are compatible with a single-file filling in $\mathbf{n p}$ channels (about 3 atoms per $\mathbf{n p}$ unit cell), and a dense stack of alternating pairs of atoms in lp channels (about 9 atoms per lp unit cell), see schematics in Fig. 1.

For a generic Langmuirian isotherm, $N(p)=N_{0} K_{\mathrm{H}} p /\left(N_{0}+K_{\mathrm{H}} p\right)$, where $N_{0}$ is the unit cell capacity and $K_{\mathrm{H}}$ is the Henry constant, the adsorption stress can be directly calculated invoking the general integral relationship between the grand thermodynamic potential and the adsorption isotherm,

$$
\Omega_{\mathrm{c}}(\mathrm{p})=-R T \int_{0}^{\mathrm{p}} N(p) \mathrm{d}(\ln \mathrm{p})=-R T N_{0} \ln \left(1+K_{\mathrm{H}} p / N_{0}\right)
$$


From equations 1 and 2, the adsorption stress as a function of the vapor pressure is expressed as

$$
\sigma_{s}(p)=R T\left\{\frac{\mathrm{d} N_{0}}{\mathrm{~d} V_{c}}\left[\ln \left(1+K_{\mathrm{H}} p / N_{0}\right)-\left(\frac{K_{\mathrm{H}} p / N_{0}}{1+K_{\mathrm{H}} p / N_{0}}\right)\right]+\frac{\mathrm{d} K_{\mathrm{H}}}{\mathrm{d} V_{c}}\left(\frac{p}{1+K_{\mathrm{H}} p / N_{0}}\right)\right\}
$$

In addition to $N_{0}$ and $K_{\mathrm{H}}$, the adsorption stress depends on the variations of these quantities with respect to the cell volume, $\mathrm{d} N_{0} / \mathrm{d} V_{c}$ and $\mathrm{d} K_{\mathrm{H}} / \mathrm{d} V_{c}$. It is noteworthy, that while $\mathrm{d} N_{0} / \mathrm{d} V_{c}$ is apparently positive, $\mathrm{d} K_{\mathrm{H}} / \mathrm{d} V_{c}$ is in general negative. This gives rise to a nonmonotonic variation of the adsorption stress and, respectively, the solvation pressure in the course of adsorption, as seen from Eq. 3. In the Henry region at low pressures, $\sigma_{s}(p) \approx R T p \mathrm{~d} K_{\mathrm{H}} / \mathrm{d} V_{c}$ is negative and decreasing, that corresponds to the sample contraction. In the saturation region at high pressures, $\sigma_{s}(p) \approx R T\left[\mathrm{~d} N_{0} / \mathrm{d} V_{c} \ln \left(K_{\mathrm{H}} p / N_{0}\right)+\mathrm{d} K_{\mathrm{H}} / \mathrm{d} V_{c}\left(N_{0} / K_{\mathrm{H}}\right)\right]$ increases and may even become positive, leading to the sample expansion. Similar nonmonotonic isotherms of solvation pressure have been calculated by using the density functional theory ${ }^{17}$ and Monte Carlo simulations ${ }^{18}$ to explain nonmonotonic deformation in the course of adsorption on zeolites and activated carbons, which is typical for many microporous materials..$^{22}$

Schematics of structure transformations upon adsorption and desorption is presented in Fig. 2. The isotherms of solvation pressure for Xe adsorption at $220 \mathrm{~K}$ in $\mathbf{l p}$ and $\mathbf{n p}$ phases of MIL-53 are given in the lower panel; the parameters employed for calculations are reported in Table 1. Since the parameters $\mathrm{d} N_{0} / \mathrm{d} V_{c}$ and $\mathrm{d} K_{\mathrm{H}} / \mathrm{d} V_{c}$ for lp and np phases as well as pre-stresses are not known, and we do not pretend here to discuss the magnitude of the adsorption deformation (which would require knowledge of the framework elasticity), the solvation pressure is given in arbitrary units, that allows us to present a qualitative scenario of structural breathing transitions observed in experiments. 


\begin{tabular}{|l|l|l|l|l|}
\hline Host structure & $K_{\mathrm{H}}$ & $N_{0}$ & $\mathrm{~d} K_{\mathrm{H}} / \mathrm{d} V_{\mathrm{c}}$ & $\mathrm{d} N_{\mathrm{o}} / \mathrm{d} V_{\mathrm{c}}$ \\
\hline lp & $50.1 \mathrm{bar}^{-1}$ & 9.0 & $-2.610^{-2} \mathrm{bar}^{-1} \AA^{-3}$ & $1.010^{-2} \AA^{-3}$ \\
\hline $\mathbf{n p}$ & $778.2 \mathrm{bar}^{-1}$ & 2.8 & $-8.26 \mathrm{bar}^{-1} \AA^{-3}$ & $4.810^{-3} \AA^{-3}$ \\
\hline
\end{tabular}

Table 1. Model parameters for adsorption of Xe in MIL-53 (Al) at $220 \mathrm{~K}$ : Langmuir parameters $\left(K_{\mathrm{H}}\right.$ and $N_{0}$ ) are taken from Ref. 12, and their derivatives with respect to unit cell volume were optimized to reproduce the experimental pressures of adsorption and desorption steps. ${ }^{12}$

According to the adopted model, adsorption starts in Ip phase. At low vapor pressures, the solvation pressure decreases causing sample contraction in an elastic fashion; the volume changes may be very minor. Once the critical value of $\sigma_{\mathrm{Ip}}^{*}$ is reached, the $\mathbf{l p}$ phase becomes unstable and the first structural transition occurs, from lp to np, causing an "abnormal breathing" - the sample contracts while inhaling. This results in a sharp uptake step on the adsorption isotherm; the sample volume decreases by $\sim 40 \%$ (line A1 in Fig. 2). Upon further increase of vapor pressure, adsorption proceeds in $\mathbf{n p}$ phase and the solvation pressure varies gradually, as is typical for the saturation region of Langmuir isotherm. At a certain point, the solvation pressure starts to increase, causing elastic expansion of the sample. Once the critical stress $\sigma_{\mathbf{n p}}^{*}$, which $\mathbf{n p}$ phase cannot withhold, is attained, a second structural transition, from $\mathbf{n p}$ to lp, occurs, causing a "normal breathing" - the sample expands while inhaling. The sample volume increases by $\sim 40 \%$. This is displayed by the second step on the adsorption isotherm (line A2). As adsorption occurs further in lp phase, the solvation pressure increases, causing elastic expansion of the sample, which remains stable.

On the desorption pass, the isotherm displays a striking hysteresis. Desorption starts and proceeds in Ip phase until the solvation pressure, which decreases as the vapor pressure decreases, reaches the critical stress $\sigma_{\mathrm{lp}}^{*}$ that triggers the structural transition from lp to np. This is displayed by a sharp downward step on the desorption isotherm (line D1). We assume here that the critical stress $\sigma_{\mathrm{lp}}^{*}$ does not depend on the adsorbed phase and is the same as that for the first $\mathbf{l p} \rightarrow \mathbf{n p}$ transition at low pressure 
adsorption. At the same time, the vapor pressure that corresponds to the critical stress $\sigma_{\text {lp }}^{*}$ exerted in lp phase in the process of desorption is lower than the vapor pressure that corresponds to the critical stress $\sigma_{\mathrm{np}}^{*}$ exerted in $\mathbf{n p}$ phase in the process of adsorption: this is the origin of hysteresis in the system under consideration. After the first desorption-induced transition, desorption proceeds further in accord with the np Langmuirian isotherm, until the solvation pressure reaches the critical stress $\sigma_{\mathrm{np}}^{*}$, leading to a final low-pressure transition back to lp phase and the second downward step on the desorption isotherm (line D2). This transition happens at a pressure lower than that of the first $\mathbf{l p} \rightarrow \mathbf{n p}$ adsorption-induced transition, explaining the low-pressure hysteresis loop, assuming again that the critical stress $\sigma_{\mathrm{np}}^{*}$ does not depend on the adsorbed phase and is determined by the mechanical properties of the $\mathbf{n p}$ framework itself.

In addition to providing a plausible stress-based mechanism for the breathing structural transitions of MIL-53 upon guest adsorption, the proposed model sheds some light into the existence of lp/np phase mixtures at pressure close to the breathing pressures. According to ref. 16, the coexistence of phases occurs to some proportion in all of the adsorption systems studied so far such as MIL-53. Thus, while no X-ray diffraction study of the Xe-loaded MIL-53 has yet been performed, the coexistence of Ip and np phases within certain pressure ranges of vapor pressures may be expected. Based on the proposed model, the simplest explanation for a possible phase coexistence can be suggested assuming that the experimental sample of MIL-53 is composed of a large number of crystallites of different sizes. If we hypothesize that the size of a given crystallite influences the critical stress that it can withhold being in the particular phase, the existence of a distribution of crystallite sizes induces a certain distribution of $\sigma_{\mathrm{np}}^{*}$ and $\sigma_{\mathrm{lp}}^{*}$ values. As such, not all crystallites will undergo phase transitions at the same vapor pressure, leading to the coexistence of the two phases within a certain pressure range. This effect causes rounding the isotherm steps in the vicinities of structural transitions. We show in Fig. 3 an example of quantitative predictions based on this hypothesis with Gaussian distributions of $\sigma_{\text {np }}^{*}$ and $\sigma_{\text {lp }}^{*}$ chosen to be compatible with the experimental spread of the adsorption and desorption steps; the upper panel 
shows the calculated isotherms, while the lower panel shows the lp-np phase composition as a function of pressure.

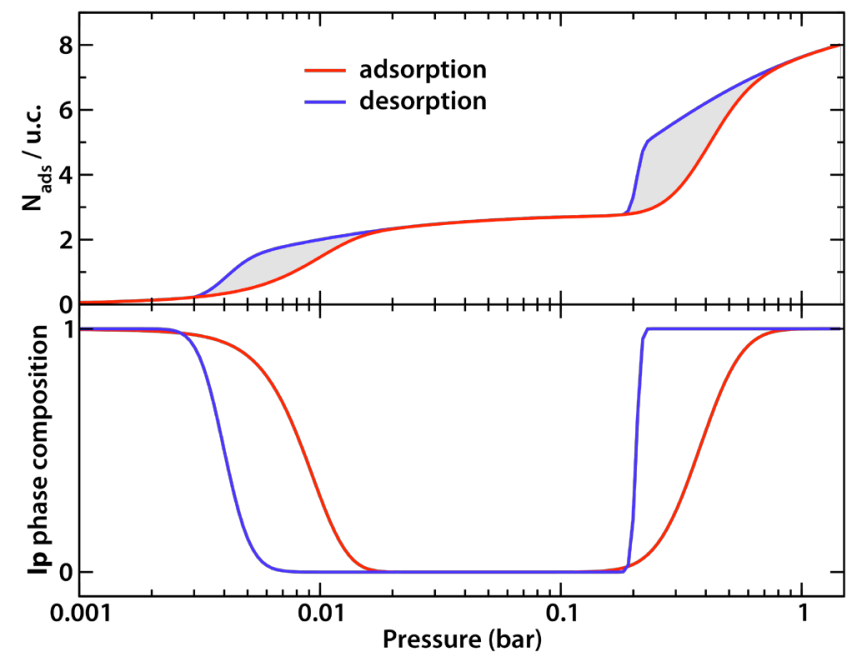

Figure 3. Calculated adsorption and desorption isotherms (upper panel) and lp phase composition (lower panel) for a model representing an assembly of crystallites of different sizes and critical stress values.

The present model can be tested against a variety of other guest-host systems at different temperatures. We also expect that this model may be applied to other types of flexible hybrid materials, such as the coordination polymers, which exhibit the so-called "gate opening" phenomenon.

ACKNOWLEDGMENT. A.V.N. acknowledges the ARO grant W911NF-09-1-0242 and the Région Île-de-France for the support via a Blaise Pascal International Research Chair, administered by the Fondation de l'École normale supérieure. 


\section{REFERENCES}

${ }^{1}$ Yaghi, O. M.; Li, H.; Davis, C.; Richardson, D.; Groy, T. L. Synthetic Strategies, Structure Patterns, and Emerging Properties in the Chemistry of Modular Porous Solids. Acc. Chem. Res. 1998, 31, 474484.

2 Kitagawa, S.; Uemura, K. Dynamic porous properties of coordination polymers inspired by hydrogen bonds. Chem. Soc. Rev. 2005, 34, 109-119.

${ }^{3}$ Férey, G.; Serre, C. Large breathing effects in three-dimensional porous hybrid matter: facts, analyses, rules and consequences. Chem. Soc. Rev. 2009, 38, 1380-1399.

${ }^{4}$ Kitagawa, S.; Kitaura, R.; Noro, S.-I. Functional Porous Coordination Polymers. Angew. Chem. Int. Ed. 2004, 43, 2334-2375.

${ }^{5}$ Ashleugh, J.; Fletchera, K; Thomas, M.; Rosseinsky, M. J. Flexibility in metal-organic framework materials: Impact on sorption properties. J. Solid State Chem. 2005, 178, 2491-2510.

${ }^{6}$ Liu, Y.; Her, J.; Dailly, A.; Ramirez-Cuesta, A. J.; Neumann, D. A.; Brown, C. M. Reversible Structural Transition in MIL-53 with Large Temperature Hysteresis. J. Am. Chem. Soc. 2008, 130, $11813-11818$.

${ }^{7}$ Li, J.-R.; Kuppler, R. J.; Zhou, H.-C. Selective gas adsorption and separation in metal-organic frameworks. Chem. Soc. Rev. 2009, 38, 1477-1504.

${ }^{8}$ Mellot-Draznieks, C.; Serre, C.; Surblé, S.; Audebrand, N.; Férey, G. Very Large Swelling in Hybrid Frameworks: A Combined Computational and Powder Diffraction Study. J. Am. Chem. Soc. 2005, 127, $16273-16278$. 
${ }^{9}$ Millange, F.; Serre, C.; Férey, G. Synthesis, structure determination and properties of MIL-53as and MIL-53ht: the first $\mathrm{Cr}^{\mathrm{III}}$ hybrid inorganic-organic microporous solids: $\mathrm{Cr}{ }^{\mathrm{III}}(\mathrm{OH}) \cdot\left\{\mathrm{O}_{2} \mathrm{C}-\mathrm{C}_{6} \mathrm{H}_{4}-\right.$ $\left.\mathrm{CO}_{2}\right\} \cdot\left\{\mathrm{HO}_{2} \mathrm{C}-\mathrm{C}_{6} \mathrm{H}_{4}-\mathrm{CO}_{2} \mathrm{H}\right\}_{x}$. Chem. Commun. 2002, 822-823.

${ }^{10}$ Loiseau, T.; Serre, C.; Huguenard, C.; Fink, G.; Taulelle, F.; Henry, M.; Bataille, T.; Férey, G. A Rationale for the Large Breathing of the Porous Aluminum Terephthalate (MIL-53) Upon Hydration. Chem. Eur. J. 2004, 10, 1373-1382.

${ }^{11}$ Serre, C.; Bourrely, S.; Vimont, A.; Ramsahye, N. A.; Maurin, G.; Llewellyn, P. L.; Daturi, M.; Filinchuk, Y.; Leynaud, O.; Barnes, P.; Férey, G. An Explanation for the Very Large Breathing Effect of a Metal-Organic Framework during $\mathrm{CO}_{2}$ Adsorption. Adv. Mater. 2007, 19, 2246-2251.

${ }^{12}$ Boutin, A.; Springuel-Huet, M.-A.; Nossov, A.; Gédéon, A.; Loiseau, T.; Volkringer, C.; Férey, G.; Coudert, F.-X.; Fuchs, A. H. Breathing Transitions in MIL-53(Al) Metal-Organic Framework Upon Xenon Adsorption. Angew. Chem. Int. Ed. 2009, 48, 8314-8317.

${ }^{13}$ Coudert, F.-X.; Jeffroy, M.; Fuchs, A. H.; Boutin, A.; Mellot-Draznieks, C. Thermodynamics of Guest-Induced Structural Transitions in Hybrid Organic-Inorganic Frameworks. J. Am. Chem. Soc. 2008, 130, 14294-14302.

${ }^{14}$ Coudert, F.-X.; Mellot-Draznieks, C.; Fuchs, A. H.; Boutin, A. Double Structural Transition in Hybrid Material MIL-53 upon Hydrocarbon Adsorption: The Thermodynamics Behind the Scenes. J. Am. Chem. Soc. 2009, 131, 3442-3443.

${ }^{15}$ Coudert, F.-X.; Mellot-Draznieks, C.; Fuchs, A. H.; Boutin, A. Prediction of Breathing and GateOpening Transitions Upon Binary Mixture Adsorption in Metal-Organic Frameworks. J. Am. Chem. Soc. 2009, 131, 11329-11331. 
${ }^{16}$ Llewellyn, P. L.; Maurin, G.; Devic, T.; Loera-Serna, S.; Rosenbach, N.; Serre, C.; Bourrelly, S.; Horcajada, P.; Filinchuk, Y.; Férey, G. Prediction of the Conditions for Breathing of Metal Organic Framework Materials Using a Combination of X-ray Powder Diffraction, Microcalorimetry, and Molecular Simulation. J. Am. Chem. Soc. 2008, 130, 12808-12814.

${ }^{17}$ Ravikovitch, P. I.; Neimark, A. V. Density Functional Theory Model of Adsorption Deformation. Langmuir 2006, 22, 10864-10868.

${ }^{18}$ Kowalczyk, P.; Ciach, A.; Neimark, A. V. Adsorption-Induced Deformation of Microporous Carbons: Pore Size Distribution Effect. Langmuir 2008, 24, 6603-6608.

${ }^{19}$ Evans, R.; Marconi, U. M. B. Phase-Equilibria and Solvation Forces for Fluids Confined Between Parallel Walls. J. Chem. Phys. 1987, 86, 7138-7148.

${ }^{20}$ Balbuena, P. B.; Berry, D.; Gubbins, K. E. Solvation Pressures for Simple Fluids in Micropores. $J$. Phys. Chem. 1993, 97, 937-943.

${ }^{21}$ Frink, L. J. D.; van Swol, F. Stress Isotherms of Porous Thin Materials: Theoretical Predictions from a Nonlocal Density Functional Theory. Langmuir 1999, 15, 3296-3301.

${ }^{22}$ Tvardovskiy, A. V. Sorbent Deformation. Elsevier, Amsterdam, 2006. 